\title{
Accomodarsi alla capacità del popolo : stategie, metodi e impatto delle missioni nel regno di Napoli, 1600-1800
}

David Gentilcore

\section{Riassunto}

David Gentilcore, «Accomodarsi alla capacità del popolo» : strategie, metodi e impatto dette missioni net Regno di Napoli, 16001800 , p. $689-722$.

Per oltre due secoli le missioni popolari costituirono un aspetto cruciale della vita religiosa, soprattutto in zone periferiche dell'Europa, dove numerosi ordini religiosi e congregazioni missionarie cercavano di tessere una rete di evangelizzazione e di istruzione catechistica. Lo studio tratta principalmente della Terra d'Otranto, provincia del Regno di Napoli (attuale Puglia meridionale), offrendo un'analisi comparativa dell'attività missionaria, sottolineando il lavoro importante ma allo stesso tempo controverso della Compagnia di Gesù. Si comincia con una discussione delle varie strategie missionarie, a cui fa seguito una messa a confronto delle tecniche e dei metodi adottati dai vari gruppi missionari per mettere in pratica le loro strategie, e si conclude con un'analisi dell'impatto che le missioni ebbero sulle popolazioni locali.

\section{Citer ce document / Cite this document :}

Gentilcore David. Accomodarsi alla capacità del popolo : stategie, metodi e impatto delle missioni nel regno di Napoli, 16001800. In: Mélanges de l'École française de Rome. Italie et Méditerranée, tome 109, n². 1997. pp. 689-722;

doi : https://doi.org/10.3406/mefr.1997.4510

https://www.persee.fr/doc/mefr_1123-9891_1997_num_109_2_4510

Fichier pdf généré le 16/09/2019 


\section{«ACCOMODARSI ALLA CAPACITÀ DEL POPOLO»: STRATEGIE, METODI E IMPATTO DELLE MISSIONI NEL REGNO DI NAPOLI, 1600-1800 ${ }^{1}$}

Mentre i missionari europei operavano nel Nuovo Mondo per attirare le anime dei "pagani" al cattolicesimo, i loro confratelli dirigevano missioni in tutta Europa. Per oltre due secoli queste missioni - chiamate interne, parrocchiali o popolari - costituirono un aspetto cruciale della vita religiosa. Numerosi ordini religiosi e congregazioni cercavano di tessere una rete di evangelizzazione e istruzione catechistica in aree dell'Europa nominalmente cattoliche ma che per molti aspetti erano rimaste tagliate fuori dal cattolicesmo ortodosso tridentino. Questo avveniva in aree particolarmente isolate ai confini dell'Europa : l'Italia meridionale costituisce un tipico esempio. Nel Regno di Napoli la persistente assenza di una efficiente struttura parrocchiale e la prevalenza di un clero alquanto mondano produsse, negli anni che sequirono la fine del Concilio di Trento (1563), un vuoto nella vita religiosa organizzata che le missioni tentarono di colmare. La mobilitazione di predicatori, confessori e istruttori fu vasta, concentrata ed incessante. Le missioni assunsero una tale importanza da essere identificate come il fenomeno "più caratteristico e importante" della storia religiosa italiana del Seicento ${ }^{2}$. Per poter esaminare la loro estensione e il loro impatto sarebbe necessario produrre una mappa della zone interessate dalle missioni delle diverse congregazioni. Ma questo approccio seriale ci direb-

${ }^{1}$ Dipartimento di Storia, Università di Leicester, Gran Bretagna.

Una versione inglese di questo saggio è apparsa sul Journal of Ecclesiastical History, 45, 2, 1994, p. 269-296; ringrazio la Cambridge University Press per il permesso di pubblicare una traduzione italiana in questa sede. Le ricerche sono state fatte mentre ero borsista alla British School at Rome, 1989-90. Ringrazio inoltre P. Francis Edwards, dell'Archivium Romanum Societatis Iesu, e Peter Burke, Giovanni Pizzorusso e Bob Scribner per i loro consigli. Per il contesto storico-religioso di questo lavoro, rimando il lettore al mio studio From bishop to witch. The system of the sacred in early modern Terra d'Otranto, Manchester, 1992.

${ }^{2}$ Carlo Ginzburg, Folklore, magia, religione, in Storia d'Italia. I : I caratteri originali, Torino, 1972, p. 603-76, a p. 656. 
be molto poco oltre al fatto che, attraverso i secoli, tutti i paesi furono visitati, la maggior parte di essi ripetutamente. Inoltre, la diversa qualità dei documenti sopravissuti porterebbe soltanto a risultati parziali. Invece, a noi interessa concoscere il modo in cui le missioni erano gestite, come erano accolte a livello locale, e se i missionari riuscirono nei loro tentativi di «evangelizzare» aree periferiche dell'Europa, perlomeno nei termini da loro stessi stabiliti.

Questo studio tratterà principalmente della Terra d'Otranto, provincia del Regno di Napoli, che corrisponde all'attuale Puglia meridionale. La scelta di un'area ristretta permette l'analisi comparativa dell'attività missionaria durante un arco di tempo relativamente lungo, e allo stesso tempo serve da "case study" in grado di aiutare a comprendere la realtà delle zone periferiche europee. Sottolineerò il lavoro della Compagnia di Gesù, in quanto può essere considerata quella che ha dato il più importante e, come vedremo, il più controverso contributo missionario nel Regno di Napoli. Inoltre, i documenti a riguardo sono di gran lunga i più dettagliati e meglio conservati, in parte per l'interesse della Compagnia a centralizzare (nel passato come nel presente). I Gesuiti erano attivi in Terra d'Otranto già nel 1573, quasi un secolo e mezzo prima che le altre congregazioni missionarie si stabilissero lì. Il loro lavoro sarà messo a confronto con quello di alcune delle congregazioni che sarebbero subentrate. Le due più attive nella $\mathrm{Pu}-$ glia meridionale furono quella dei Padri della Missione, fondata da S. Vincenzo de' Paoli (chiamati anche Lazzaristi o Vincenziani), presente in Terra d'Otranto dal 1729, e la Congregazione del SS.mo Redentore, fondata da S. Alfonso de' Liguori nel 1722 (i Redentoristi). Si farà riferimento anche alla Congregazione della Missione apostolica, fondata nel 1646 con base a Napoli. Comincerò con l'esaminare le strategie delle varie Congregazioni missionarie, a cui farà seguito una messa a confronto delle tecniche e dei metodi da essi adottati per mettere in practica le loro strategie. Il paragrafo finale tratterà dell'impatto che le missioni ebbero sulle popolazioni locali, così come delle critiche ad esse rivolte e dell'influenza che queste ebbero nella riforma delle tecniche missionarie.

Molti temi caratterizzano i tentativi dei missionari di «evangelizzare» (per adottare il termine più usato dagli stessi missionari) $i$ "pagani " a loro più prossimi. Il più evidente risulta essere il messaggio religioso in se stesso, semplificato e insistente, miracolistico e spaventoso. La sua efficacia dipendeva dalla intensa serie di prediche e processioni, e da un altrettanto frequente ricorso alle confessioni e alle pacificazioni, sia quelle messe in scena sia quelle spontanee. Il successo di questo messaggio non dovrebbe essere sottovalutato, nonostante sia evidente che poteva essere frainteso o foggiato in modo che si adattasse alla mentalità popolare. Collegati a que- 
sto sono gli oggetti stessi delle missioni, descritti dai missionari come "rurali» o "urabani», "ignoranti» o "civilizzati», e le tensioni tra il missionario e i destinatari della missione, tra il centro e la periferia. Naturalmente questi temi non riguardavano solo le missioni europee, come dimostra il frequente riferimento dei missionari alle "Indie», e si farà cenno a sviluppi analoghi in Asia e nelle Americhe.

Per cominciare, diamo uno sguardo alla strategia missionaria dei Gesuiti. I Gesuiti con le loro missioni non cercarono di sviluppare e far radicare una religione di élite, ma una religione che fosse accessibile e comprensibile a tutti. L'importanza data dai Gesuiti all'intervento miracoloso dei santi, al potere delle reliquie, alle devozioni e alle penitenze, costituiva parte di questa strategia (anche se potremmo considerarla piuttosto come un impedimento allo sradicamento della "superstizione") ${ }^{3}$. Per "conquistare» le masse, i Gesuiti contavano sulle prediche e sul potere evocativo del liguaggio e dei gesti, dando particolare importanza all'affettività e all'emotività per fare appello all'immaginazione dei fedeli e condurli alla conversione ${ }^{4}$. Tale approccio avrebbe prodotto più tardi opposizione verso la loro attività missionaria e nello stesso tempo avrebbe condizionato le strategie adottate dalle altre congregazioni missionarie. La forma di missione popolare più comunemente praticata dai Gesuiti era la missione centrale o segneriana, così chiamata perché fu ideata dal Gesuita modenese Paolo Segneri senior (1624-94) $)^{5}$. Questa missione comportava la scelta di una località urbana che permettesse sia la partecipazione della gente del pease sia della campagna circostante. Generalmente durava da otto a dieci giorni, dopo di chè i missionari si spostavano in un'altra località vicina. Per provocare il pentimento facevano affidamento sull'intensità e sulla straordinarietà che l'evento assumeva nella vita della popolazione del luogo. A fare da complemento c'era la missione parrocchiale, che durava parecchi giorni e vedeva i missionari andare da un villaggio all'altro, seguendo un itinerario

${ }^{3}$ Ibidem, p. 152.

${ }^{4}$ Paola Vismara Chiappa, Forme della pietà barocca nelle campagne lombarde tra Sei e Settecento, in A. De Maddalena, E. Rotelli, G. Barbarisi (a cura di), Economia, istituzioni, cultura in Lombardia nell'età di Maria Teresa, II : Cultura e società, Bologna, 1982, p. 815-16.

${ }^{5}$ Per biografie dei missionari gesuiti attivi nella penisola italiana, cfr. Armando Guidetti, Le missioni popolari. I grandi Gesuiti italiani, Milano, 1988. Per il Settecento, cfr. l'articolo (con appendici) di Giuseppe Orlandi, S. Alfonso Maria de' Liguori e l'ambiente missionario napoletano del Settecento : la Compagnia di Gesù, in Spicilegium historicum Congregationis SS.mi Redemptoris (d'ora innanzi : SHCSR), XXXVIII, 1990, p. 5-195. 
di lavoro che attraversava tutta la campagna. Queste missioni venivano chiamate "scorrerie" per la loro breve durata ${ }^{6}$.

Nei documenti sopravvissuti le missioni centrali sono descritte dettagliatamente, mentre le missioni parrocchiali lo sono di meno, forse perchè costituivano, più che altro, un lavoro di routine, ripetitivo nello stile $\mathrm{e}$ nella struttura. Se mai vengono menzionate, questo avviene generalmente nelle litterae annuae che contengono una sintesi degli avvenimenti e dei risultati degni di nota, selezionati dalle varie relazioni mandate da ciascun Collegio del Regno. Le litterae annuae sono più generiche in quanto citano risultati e avvenimenti alquanto stereotipici, e nello stesso tempo sono più specifiche delle cosiddette "relazioni" missionarie, perché non cercano di raccontare la missione in se stessa ma uno o due avvenimenti salienti? ${ }^{7}$.

Le missioni centrali invece tenevano conto delle elaborate processioni penitenziali e degli appassionati sermoni, degli atti di pacificazione rappresentati e della conversione di peccatori ostinati dinanzi a folle numerose radunate nella cattedrale o nella piazza centrale, come vedremo in seguito. Tutto questo si adattava perfettamente allo stile dettagliato, vivace, retorico e trionfalistico delle relazioni che i missionari scrivevano. Queste relazioni dovevano servire sia da modello e da fonte di ispirazione per le missioni future, sia da testimonianza per i posteri dei trionfi e dei successi riportati dai missionari Gesuiti a gloria dell'intera Compagnia. Risulta anche chiaro che le relazioni pubblicate, di cui ogni missionario era a conoscenza, tendevano a influenzare il modo in cui altre missioni venivano percepite e registrate, anche nel momento in cui avevano luogo. Per questa ragione le relazioni contengono molti stereotipi e topoi, come per esempio, l'ignoranza e la superstizione delle masse rurali. Regioni come l'Italia

- Per un descrizione della procedura missionaria gesuitica, cfr. Elisa Novi Chavarria, L'attività missionaria dei Gesuiti nel Mezzogiomo d'Italia tra XVI e XVIII secolo, in G. Galasso e C. Russo (a cura di), Per la storia sociale e religiosa del Mezzogiorno d'Italia, II, Napoli, 1982, p. 159-85. Per uno studio delle varie Congregazioni presenti a Napoli, cfr. Maria Gabriella Rienzo, Il processo di cristianizzazione e le missioni popolari nel Mezzogiorno. Aspetti istituzionali e socio-religiosi, in ibidem, I, p. 440-81. Per uno studio dei Gesuiti in Puglia, cfr. Mario Rosa, Strategia missionaria gesuitica in Puglia agli inizi del Seicento, in Id., Religione e società tra Cinque e Seicento, Bari, 1976, p. 245-72.

${ }^{7}$ Per il 1649 si possono confrontare la relazione della missione fatta a Lecce e il riferimento alla missione nella serie di litterae annuae; normalmente se fra i documenti è presente la relazione di una missione questa prende il posto di un riferimento nelle lettere annuali. Cfr. Archivium Romanum Societatis Iesu (d'ora innanzi : ARSI), Provincia Neapolitana, 74, XXVI, e ibidem, XXVII, f. 251-252v. and 254. 
meridionale, l'Andalusia, il Delfinato e parti della Bretagna erano identificate come "le Indie», un concetto questo che serviva a dare risalto ai "successi» del missionario. Questo topos aveva le sue origini nei tentativi di trasferire l'impulso da crociata dell'attività missionaria extraeuropea nelle regioni interne dell'Europa. Così, quando il missionario e predicatore S. Francesco de Geronimo (1642-1716) chiese ai suoi superiori Gesuiti di essere mandato in Giappone per dare la sua vita per le fede, gli fu risposto che, invece, "era buono per far l'Apostolo nell'India di questa Città e Regno di Napoli ${ }^{\sharp}$ ". La prevalenza di un tale topos non permetteva ai missionari di valutare chiaramente le situazioni particolari con cui avevano a che fare? Le religioni non cristiane dei «pagani» del Nuovo Mondo e le credenze «superstiziose » dei contadini europei erano comparabili, dal momento che entrambe venivano poste alla stessa estremità dello spettro religioso, opposte alla vera fede. Entrambe richiedevano un'opera di evangelizzazione. Sia nel Nuovo Mondo che nel Vecchio la "colonizzazione culturale ${ }^{10}$ " dei Gesuiti voleva essere sia evangelizzante che civilizzante. Per ciò, la fondazione delle congregazioni mariane per i laici fu accompagnata dalla fondazione di collegi per la formazione delle élites locali. L'impeto da crociata che caratterizzava le missioni nel Nuovo Mondo fu trasferito nelle missioni interne europee. Le relazioni riflettono questa ferma convinzione. Stando a queste i missionari non potevano sbagliare, e le loro fatiche erano destinate a produrre sempre dei frutti, qualunque fosse l'ostacolo da superare. Anche se questo loro atteggiamento può distorcere $\mathrm{i}$ «fatti " della missione, corrisponde all'opinione che i missionari avevano dell'importanza della loro attività. Innanzitutto, si doveva dimostrare che la presenza dei missionari godeva dell'approvazione divina. I numerosi miracoli verificatisi grazie alle devozioni da loro introdotte - specialmente quelle dedicate all' "Apostolo delle Indie»S. Francesco Saverio - lo testimoniavano. Lo stesso valore di testimonianza veniva attribuito alle visioni avute dalla gente del paese che preannunciavano la venuta dei missionari prima ancora che qualcuno potesse sapere del loro imminente arrivo. La nevicata che si verificò durante

${ }^{8}$ Archivio Segreto Vaticano (d'ora innanzi : ASV), Congregazione dei Riti, no. 2022, f. 232.

${ }^{9}$ Cfr. Adriano Prosperi, "Otras Indias": Missionari della Controriforma tra contadini e selvaggi, in Scienze, credenze occulte, livelli di cultura. Convegno internazionale di studi, Firenze, 1982, p. 206-34, a p. 217-18; e Jean Delumeau, La storia della cristianizzazione, in Id., Cristianità e cristianizzazione. Un itinerario storico, Casale Monferrato, 1983, p. 177.

${ }^{10}$ L'espressione è usata da Alain Croix per descrivere le missioni dei Gesuiti nella Bretagna rurale; cfr. La Bretagne aux XVI et XVII' siècles. La vie, la mort, la foi, II, Parigi, 1981, 1242. 
una missione nell'estate del 1650 fu interpretata come un segno divino che ne preannunciava il successo".

Le varie altre congregazioni missionarie attive nella penisola italiana svilupparono ciascuna il proprio orientamento e la propria strategia cercando di tener conto di alcune delle critiche fatte ai Gesuiti. L'opposizione verso l'aspetto emozionale e teatrale delle devozioni dei Gesuiti e verso il carattere spaventevole dei loro sermoni sarà esaminato ulteriormente nel paragrafo finale. Nel 1746 il frate e missionario di formazione gesuitica S. Leonardo da Porto Maurizio (1676-1751) scrisse al vescovo di Ferrara informandolo sulle varie forme di missione allora in atto. In questa lettera non condanna le missioni gesuitiche principalmente per le loro devozioni esteriorizzate, ma per la loro durata relativamente breve :

Due modi di Missionare osservo nella Chiesa di Dio : Il primo è dei P.P. della Compagnia [di Gesù], ed è fuoco con molte processioni ed esteriorità : il secondo è dei P.P. Missionari di S. Vincenzo, tutto quiete ed esclude ogni sorta di esteriorità; ambidue son fruttuosi, eppure io che giro il mondo, ho toccato con mani che il secondo modo è molto più fruttuoso che il primo [che] non dura che otto, o dieci giorni, e non più, nè si dà tempo di sciogliere tutti i nodi nel Confessionale... Al contrario nel secondo modo di Missionare si lavora nel Confessionale, ed ogni Missione durerà un mese, e si quietano le coscienze... Con tutto ciò, io ho giudicato prendere una via di mezzo; mi servo dell'esteriorità de' Gesuiti, ma con moderazione, e riprovo la brevità del tempo' ${ }^{12}$.

I Lazzaristi fondarono la loro prima comunità religiosa in Italia nel 1642 a Roma, ma fu solo nel 1729 che riuscirono a stabilirsi a Oria in Terra d'Otranto. I missionari, molti dei quali piemontesi, diedero risalto all'elemento catechetico e, oltre a insegnare teologia morale e a condurre esercizi spirituali presso la loro residenza, furono presto chiamati a dirigere il seminario di Oria. Nel 1732 si stabilirono a Lecce e, nonostante le difficoltà finanziarie che questa ulteriore espansione comportava, riuscirono a completare la costruzione della loro residenza nel $1743^{13}$. Così come a Oria, anche qui i Lazzaristi si dedicarono sia al lavoro della missione ${ }^{14}$ sia all'inse-

"ARSI, Neap., 75, ii, f. 21.

${ }^{12}$ S. Leonardo da Porto Maurizio, Opere complete, IV, Venezia, 1868, p. 556-59; cit. in Giuseppe Orlandi, La missione popolare redentorista in Italia. Dal Settecento ai giomi nostri, in SHCSR, XXXIII, 1985, p. 51-141, a p. 59.

${ }^{13}$ Pompeo Silva, Cenni storici sulla Congregazione della Missione in Italia (16421925), Piacenza, 1925, 170-8.

${ }^{14}$ Archivio della Casa della Missione, Lecce, Libro in cui si notano le Missioni fatte dalla Casa della Missione di Lecce, vol. I, 1733-1902. 
gnamento degli ordinandi e alla gestione dei seminari diocesani che venne loro affidata nel 1740. Le prediche dei missionari Lazzaristi trattavano i tipici temi barocchi della paura della morte, della collera divina e del rischio di una finale impenitenza. Eppure la semplicità delle loro prediche è in stridente contrasto con la teatralità addottata dai Gesuiti. I predicatori Lazzaristi cercavano di essere "popolari» nel loro modo di esporre, per assicurarsi la comprensione di tutti i fedeli.

Esser molto popolare - si consigliava negli "Ordini per quelli che vanno in Missione" - cioè accomodarsi alla capacità del popolo, lasciando le sottigliezze per appigliarsi al frutto, ed all'utile, discendendo al particolare, e servendosi sempre del nostro ordinario metodo, non scordandosi sopratutto di questi quattro ponti in ogni predica : $1^{\circ}$ dichiarare e spiegare con chiarezza quello che vorrà persuadere al popolo; $2^{\circ}$ dirne li motivi più sensibili e proporzionati alla capacità di coloro, a quali si parla; $3^{\circ}$ dar mezzi particolari e facili per acquistare o far tal cosa; $4^{\circ}$ non tralasciar mai di prevenire l'obiezioni che potrebbero farsi, ne dir mai niente d'indigesto, che possa lasciar qualche errore o dubbio alla gente semplice ${ }^{15}$.

I Redentoristi di S. Alfonso de' Liguori cercarono di mettere insieme i due stili missionari - penitenziale e catechistico - per addottare una strategia ritenuta più idonea alle masse rurali dell'Italia meridionale. Così, anche se il loro modo di predicare risultava emotivo e teatrale proprio come quello dei Gesuiti, Alfonso de' Liguori volle che fosse seguito da pie devozioni e funzioni in modo che portasse a conversioni durevoli. La strategia dei Redentoristi, più di quella dei Gesuiti, tendeva a essere popolareggiante, per andare incontro alla cultura popolare. La loro devozione influenzava ed era nello stesso tempo influenzata dai modi locali di concepire il sacro, un mondo che Alfonso de' Liguori conosceva intimamente. Lui stesso volle assicurarsi che i Redentoristi conducessero le missioni soprattutto nelle zone rurali, che lui riteneva fornissero un terreno più fertile che le città. In parte questo concetto rappresentava una reazione verso la missione centrale dei Gesuiti dimostrato dalle élites cittadine, come vedremo nel paragrafo finale. Nasceva anche da un generalizzato avvicinamento ecclesiale alla campagna. Durante il diciottesimo secolo, in netto contrasto con il diciassettesimo, non è più «la città che deve unificare con la sua religione il disperso, sospetto e temuto mondo delle campagne : al contrario, è la religione delle

${ }^{15}$ Archivio del Collegio Leoniano, Roma, Ordini per quelli che vanno in Missione, f. 17v., cit. in Luigi Mezzadri, Le missioni popolari della Congregazione della Missione nello Stato della Chiesa (1642-1700), in Rivista di storia della Chiesa in Italia, XXXIII, 1979, p. 12-44, a p. 35. 
campagne che deve essere tutelata e proposta a modello alle città in via di progressiva scristianizzazione ${ }^{16}{ }^{\prime}$.

Differenti strategie missionarie produssero differenti metodi e tecniche di attuazione. Soffermiamoci ora su questi, e sull'introduzione di alcune devozioni, cominciando dai Gesuiti e continuando poi con le reazioni e gli adattamenti delle altre congregazioni. La missione svoltasi a Lecce nel 1639 costituisce un tipico esempio di missione centrale gesuitica ${ }^{17}$. Il sabato pomeriggio precedente, venivano affissi gli avvisi che annunciavano la missione. Missionari in processione con stendardo e crocifisso invitavano la gente a radunarsi la domenica successiva nel palazzo vescovile per l'inizio della missione. La mattina dopo veniva esposto il SS.mo Sacramento nelle tre parrocchie della città. L'esposizione veniva ripetuta nel pomeriggio nel palazzo vescovile (dove sarebbe stato esposto ogni pomeriggio della missione), ed era accompagnata da una predica. Dopo di che, il Sacramento veniva portato in processione per la città, con a capo il sindaco con lo stendardo della città e la nobiltà, seguiti dal rettore del collegio dei Gesuiti che portava un crocifisso ed era accompagnato da quattro preti, che reggevano torce accese, e dalle confraternite della città. Alla fine c'era il clero con il SS.mo Sacramento. Le attività della missione cominciavano all'alba di ogni giorno della settimana successiva, con l'esposizione del SS.mo Sacramento e le prediche per $\mathrm{i}$ lavoratori. A ciò seguiva la predica nelle parrocchie, dopo di che il SS.mo Sacramento veniva riportato in processione al palazzo vescovile. Ogni mattina arrivavano processioni di gente in abito di penitenza dai paesi vicini, dirette verso il palazzo vescovile. Nel pomeriggio veniva impartito l'insegnamento dei principi elementari della dottrina cristiana ai bambini che si preparavano per la prima comunione. La sera, dopo i vespri, veniva impartito un insegnamento generale, accompagnato da meditazioni e preghiere. Le confessioni venivano ascoltate per tutto il giorno. La notte, processioni composte da preti con crocifisso e torce e da membri delle confraternite che reggevano teschi e cantavano il Miserere, partivano dal collegio e si snodavano in varie zone malfamate della città, esortando i peccatori a pentirsi. La gente che era presente alla predica veniva poi invitata a seguire la processione che tornava alla chiesa dei Gesuiti, all'esterno della quale veniva fatta un'altra predica. Dopo gli uomini entravano per partecipare agli esercizi penitenziali. La mattina dell'ultimo giorno, una domenica, veniva celebrata la messa principale della missione. Nel

\footnotetext{
${ }^{16}$ Adriano Propseri, Il missionario, in Rosario Villari (a cura di), L'uomo barocco, Roma, 1991, p. 179-218, a p. 216.

${ }^{17}$ ARSI, Neap., 73, xliv, ff. 453-5.
} 
pomeriggio veniva fatta una predica seguita dalla benedizione pontificale. La missione si chiudeva con un'ultima processione.

I Redentoristi seguirono la tradizione missionaria dei Gesuiti cercando, nello stesso tempo, di porre rimedio alla mancanza di missioni penitenziali. Introdussero, per esempio, particolari «funzioni», come gli esercizi della Vita Devota : mezz'ora di insegnamento metodico sulla preghiera, preparazione e ringraziamento per la comunione, a cui seguiva una spiegazione della "Regola di vita del cristiano devoto» e che si concludeva con una breve meditazione sulla Passione o sui dolori della Vergine Maria ${ }^{18}$. Pur avendo apportato tali innovazioni, i Redentoristi adottarono molte pratiche dei Gesuiti e le arricchirono. Un esempio è costituito dai "sentimenti di notte», esortazioni serali che consistevano di una processione che partiva dalla chiesa dopo la predica serale, con torce e crocifisso, canto di inni, e giungeva nella piazza principale dove si teneva un breve «fervorino». Dopo di che, la processione ritornava verso la chiesa. Per prolungare ulteriormente l'impatto della missione, i missionari redentoristi ritornavano quattro o cinque mesi dopo per condurre un "esercizio di rinnovamento spirituale», della durata di alcuni giorni, che consisteva di confessioni, brevi prediche, insegnamento e consigli di carattere spirituale.

Come le altre congregazioni, anche i Redentoristi insegnavano che bisognava confessarsi in modo profondo e completo e poi ricevere l'eucarestia, con la speranza che ciò diventasse un'abitudine regolare. Come scrisse il missionario Vincenzo Gagliardi : «Essendo il fine della missione di rimettere tutte le anime in grazia di Dio e per la diritta strada della vita eterna, e perciò altro non deve pretendersi colla missione che una buona confessione ed una ferverosa comunione ${ }^{19}$ ". I missionari insistevano molto sulla confessione generale, poiché erano consapevoli del grande numero di confessioni «sacrileghe» (cioè incomplete) fatte ai parroci. Anche queste erano spesso poco frequenti, fatte, in molti casi, solo sul letto di morte. Questa diffusa resistenza alla confessione tra i fedeli può essere attribuita non tanto ad indifferenza religiosa quanto alla difficolta insita

18 Samuel Boland, The missionary methods of the Redemptorists, in SHCSR, XXX, 1982, p. 401-47, a p. 409.

19 Vincenzo Gagliardi, Direttorio Apostolico o sia Metodo di Missione in cui ci sono gli esercizj da farsi in essa per bene delle anime, 1806, f. 156. Manoscritto curato e presentato da Giuseppe Orlandi, in SHCSR, XXX, 1982, p. 5-289. Il volume piccolo $\left(16^{\circ}\right)$ ma irrobustito da una copertina di cuoio fu probabilmente portato da paese in paese, dove il missionario poteva spesso trovarsi senza altri libri per preparare la predica. Gagliardi lo scrisse in una calligrafia miniscola per rispiarmare carta e rendere il volumetto più facilmente trasportabile. 
nel fatto di dover confessare i propri peccati al parroco, un membro della stessa comunità. Il missionario, essendo un estraneo, sconosciuto alla gente del paese e presente nella comunità solo temporaneamente, non creava lo stesso problema psicologico ${ }^{20}$. Inoltre la sua preparazione, la sua istruzione ed esperienza nel confessionale, lo mettevano certamente in una posizione migliore di quella di un comune parroco del periodo. L'insistenza sulla confessione frequente, adottata per la prima volta dai Gesuiti, era destinata a diventare una caratteristica comune a tutte le strategie missionarie. Non era soltanto una parte importante della pietà barocca, ma offriva anche un segno tangibile di "conversione» durante il corso della missione, e la sua pratica abituale era un mezzo per prolungare gli effetti della missione. L'importanza data alla confessione durante la missione era tale da prevalere finanche sull'istruzione religiosa, basilare come'era. La stessa cosa valeva per tutte le congregazioni missionarie. L'istruzione catechistica veniva considerata dai missionari non come fine a se stessa ma come uno strumento per raggiungere una fine : "sì per la buona confessione che è l'effetto della santa missione, sì per rimediare alle confessioni nulle e sacrileghe passate», come scrisse il redentorista Gagliardi ${ }^{21}$. Allo stesso modo, le lezioni di dottrina cattolica impartite dai missionari gesuiti avevano come punto focale il peccato e la necessità della confessione. Gli argomenti delle dieci lezioni (all'incirca una al giorno) erano i seguenti :

1) Integrità della confessione; 2) Sopra il dolore necessario alla confessione; 3) Sopra il proposito necessario alla confessione; 4) Sopra l'obbligo della restituzione, e della limosina; 5) Sopra il battesimo, e il matrimonio; 6) Sopra i peccati più gravi della lingua; 7) Sopra la gravezza del peccato mortale; 8) Sopra la passione del odio, e del amore; 9) Sopra la gravezza dei peccati disonesti; 10) Sopra i rimedi per non cadere in peccato ${ }^{22}$.

In termini generali, la missione lazzarista era molto più semplice e meno sensazionale di quella dei Redentoristi o dei Gesuiti. Aveva tre caratteristiche : una predica la mattina presto prima che la gente partisse per andare al lavoro, il "piccolo catechismo" per i bambini nel pomeriggio, e catechismo per tutti la sera. La differenza con la missione gesuitiça stava nella sua lunghezza : un minimo di due settimane nelle comunità più piccole $e$ fino a un mese in quelle più grandi, cosa che permetteva l'accurata confessione di tutta la popolazione. Inoltre, i resoconti delle loro missioni cerca-

${ }^{20}$ Delumeau, Missioni "interne» nel XVII secolo, in Cristianità, cit., p. 206-9.

${ }^{21}$ Gagliardi, Direttorio, cit., f. 134.

${ }^{22}$ Giovanni Pietro Pinamonti, Istruzioni per le Missioni, ARSI, Opera nostrorum, p. 225. 
vano di essere relativamente oggettivi. Contengono pochi riferimenti a miracoli successi durante le missioni, o alla giustificazione divina delle attività dei missionari (in contrasto con le relazioni delle missioni gesuitiche), e giudicavano le risposte alle missioni o come «molto ferventi», "ferventi» 0 «fredde ${ }^{23}$ ».

Tutte le congregazioni facevano affidamento, come parte del loro metodo missionario, sull'introduzione di nuove devozioni o sull'ulteriore diffusione di quelle già esistenti. I Gesuiti mettevano in risalto devozioni radicate come il rosario, "cosa che hà cagionato grand'affetto alla Compagnia nell'Ordine de' Predicatori ", come raccontò una relazione ${ }^{24}$. In questo modo cercavano di completare piuttosto che sostituire quell'attività pastorale che già esisteva e di evitare di alienarsi altri membri del clero. Incoraggiarono devozioni tipiche della Controriforma come il culto dell'angelo custode, della buona morte e del loro S. Francesco Saverio. Altre devozioni rispecchiavano la strategia gesuitica dell' «accomodamento». Questa consisteva nel sostituire pratiche da loro ritenute eterodosse - il risultato di ciò che essi definivano ignoranza o superstitizione - con pratiche più ortodos$\mathrm{se}^{25}$. In questo modo le feste del carnevale, del calendimaggio, della notte di S. Giovanni e altre ancora, considerate scandalose ed empie, venivano sostituite da processioni e rappresentazioni religiose, e da esercizi di devozione come le quarantore. Tramite queste sostituzioni i Gesuiti tentavano di mettere in pratica le riforme tridentine, togliendo l'iniziativa religiosa ai laici. La prima volta che le quarantore furono "rappresentate" durante il carnevale fu a Macerata nel 1556, per allontanare i cittadini benpensanti da una commedia carnevalesca che i Gesuiti avevano descritto come impura e oscena. Per realizzare il loro scopo esposero il SS.mo Sacramento «con un bello e inusitato apparato di lumi e di addobbi ${ }^{26}$ ». Tale fu il suo successo

${ }^{23}$ Mezzadri, Missioni, cit., p. 21.

${ }^{24}$ ARSI, Neap., 76 I, iv, f. 28.

${ }^{25}$ Nelle missioni d'oltremare l' «accomodamento" portava i missionari anche a vestire abiti e ad adottare costumi locali, ma soltanto in quelle società che erano considerate avanzate e dove la religione era ritenuta senza idolatria, come in Cina. Qui le missioni erano riservate alle sole classi dirigenti (per un esempio di questa strategia, cfr. Jonathan Spence, The Memory Palace of Matteo Ricci, Londra, 1984). Le strategie messe in pratica nelle missioni europee, al contrario, assomigliavano molto di più a quelle adoperate nelle Americhe, dove gli Indios erano ritenuti idolatri e meno avanzati. Secondo questa categorizzazione, il "selvaggio" americano era uguale al "contadino" europeo. Cfr. Propseri, Il missionario, op. cit., p. 203.

${ }^{26}$ Nicolò Orlandini, Historiae Societatis Iesu prima pars, Roma, 1615, p. 540; cit. in Pietro Tacchi Venturi, Storia della Compagnia di Gesù in Italia, narrata col sussidio di fonti inedite, I, Roma, 1950, p. 240-1. 
che alla fine del secolo la devozione veniva praticata in tutti i collegi e le residenze dei Gesuiti durante il carnevale. Un altra devozione gesuitica ma allo stesso tempo tipicamente barocca, era l'atto di umiltà chiamato "lo strascino": il penitente si inginocchiava, abbassava la testa e strisciava la lingua per terra in segno di mortificazione. I missionari si resero conto che nuove devozoni come questa avrebbero avuto maggior successo se, quando venivano introdotte fossero state praticate o adottate da persone autorevoli del luogo. Perciò la relazione di una missione del 1669 nella cittadina di Putignano riporta con soddisfazione che il vicario, "conoscendo che l'attione per essere più esemplare, era maggiormente meritoria, ... volea col grido di tal publica mortificazione ridurne qualche pecorella smarrita all'ovile di Christo». Insieme a molti componenti del clero locale, dopo la predica e gli atti di penitenza fece lo strascino, «strascinando la lingua per terra, dalla porta della Chiesa sin all'altare maggiore, [volendo] in tal modo venire à riconoscere il suo creatore, con cercarli perdono delle sue offese ${ }^{27}$ ".

Ai Gesuiti si deve anche la formazione degli «Schiavi della Vergine Marian, una confraternita ad hoc che consisteva di sette uomini scelti tra gli abitanti del paese. In una tipica devozione gli "schiavi» andavano in processione, incatenati, il primo reggendo un crocifisso, fino alla chiesa parrocchiale. In chiesa, si prostravano di fronte all'altare maggiore chiedendo perdono per i loro peccati e consacrando la loro vita alla Regina del cielo.

Per introdurre divotione magiore verso la Vergine nostra Signora, si è procurato in ogni terra, se bene in diverse maniere, fare alcuni schiavi della Vergine, e Sacerdoti, e Gentilhuomini, e plebei, i quali servissero come tanti specchi al resto del Popolo; non si può credere con che desiderio, e fame desiderava ciascheduno si gloriosa catena ${ }^{28}$.

Gli schiavi della Vergine portavano a reazioni immediate di grande fervore, anche se è difficile stabilire la durata di questo stato. Per esempio, secondo la relazione della missione del 1666 appena citata, alcuni chierici "s'essibirono à digiunare per molti sabbati, altri à dire il sabbato la messa della Vergine, alcuni giovanetti del Clero si vollero tagliare le chiome, al cui esempio tutto il Clero della Terra [di Galatina] riformò i suoi Capelli, et altri à diverse limosine in agiuto de' poveri ${ }^{29}$ ». Questa devozione poteva assumere forme "carnevalesche", non solo perché vedeva coinvolti ricchi e poveri insieme ma anche perché si faceva uso delle maschere. Nel paese di Parabita «i padroni del luogo vollero essere schiavi della Vergine nella ca-

\footnotetext{
${ }^{27}$ ARSI, Neap., 76 I, xviii, f. 96-7.

${ }^{28}$ ARSI, Neap., 76 I, iv, f. 27.

${ }^{29}$ Ibidem, f. $27 \mathrm{v}$.
} 
tena dei Plebei. Il simile hà fatto il Duca di Seclì, et un cavaliero in Matino, che vestitosi anco da Villano con una Croce in ispalla volle procedere i suoi terrazzini ${ }^{30}$ ". Il fatto che elementi carnevaleschi avessero un ruolo così importante in queste devozioni potrebbe sembrare in un certo qualmodo ironico se si considera la volontà dei missionari di eliminare il Carnevale. Ma in pratica questo faceva parte della strategia dell'accomodamento, a cui si è fatto riferimento sopra. In questo senso si potrebbe parlare della cristianizzazione del carnevalesco, anche se gli oppositori dei Gesuiti in quel tempo erano meno caritatevoli.

L'elemento carnevalesco è evidente specialmente in tutti i numerosi resoconti delle processioni penitenziali. Queste processioni diventarono uno degli aspetti più controversi della missione gesuitica. Una processione tipica era una vera messa in scena, che includeva non solo flagellanti ma anche gente travestita da santi. Poteva anche prendere la forma della processione che si snodava dal paese di Surbo per incontrare la missione urbana a Lecce, "con una bella rappresentatione della sacra passione con personaggi vivi, e molti battenti ${ }^{31}$ ». Una processione del 1646 che attraversò il paese di Squinzano deve essere stato uno spettacolo proprio raccapricciante : vi era radunato tutto il clero, la nobiltà e il popolo del paese, scalzi e coperti di ceneri, legati insieme con catene e corde come prigionieri, secondo l'analogia riportata dall'autore della relazione. Un barone locale si colpiva con una pietra, aiutato da suo figlio in abito francescano. Una prostituta pentita era travestita da Maria Maddalena, con un abito di sacco e con un "chiappo" appeso al collo, e si batteva il petto con una pietra. Un'eremita, travestito da S. Giovanni, si flagellava ${ }^{32}$. La teatralità non era limitata alle processioni penitenziali. In una processione che partiva da un paesino per partecipare all'Eucarestia nel corso di una missione centrale in un altro paese, "tutte le Donne maritate coronate di spine co' Bambini in braccio parimente coronati come tante statue della Vergine ${ }^{33}$ ". Il travestimento era usato dai Gesuiti per inculcare nei fedeli l'importanza della prima comunione. I ragazzi venivano vestiti come angeli, e portavano in mano ghirlande di alloro e di palma, mentre le ragazze «rapresentavano diverse Vergini, tra quali altre sotto la bandiera di S. Ursula da una rapresentata, altre mostravano i misteri di S. Apollonia, S. Lucia, S. Catarina Vergine e Martire, et altre innumerabili ${ }^{34}$ ».

\footnotetext{
${ }^{30} \mathrm{Ibidem}$.

${ }^{31}$ ARSI, Neap., 73, xliv, f. 454v.

${ }^{32}$ ARSI, Neap., 74, xvi, f. 215.

${ }^{33}$ ARSI, Neap., 76 I, iv, f. 27.

${ }^{34}$ Ibidem, xviii, f. $108 v$.
} 
Un altro aspetto importante della missione era quello che i Gesuiti chiamavano "lo spettacolo del perdono universale», e che consisteva nella celebrazione pubblica di un atto di pacificazione tra due parti in disputa tra loro. Lo scopo era quello di incoraggiare i partecipanti a perdonare a turno coloro che li avevano offesi, con i missionari nelle vesti di esperti mediatori ${ }^{35}$. Si trattava di una applicazione dell'arbitrato medioevale, una forma di giustizia spesso usata per porre fine a faide e vendette private così comuni nelle società "faccia a faccia». L'arbitrato integrava l'attività dei tribunali regolari e si basava sulla libera scelta dei giudici da parte dei due interessati, a cui faceva seguito una pubblica cerimonia di pacificazione. Tutto veniva verbalizzato da un notaio. L'atto di riconciliazione era reso molto coreografico e veniva preparato in precedenza, inclusi i discorsi che dovevano essere recitati da entrambe le parti in causa ${ }^{36}$. Per i missionari gesuiti l'importanza di queste pacificazioni era tale che gerneralmente gli riservavano un paragrafo a parte nelle relazioni. Si tenevano in chiesa, dopo la predica, quando si baciavano i piedi di Cristo crocifisso. Se il caso lo richiedeva, entrambe le parti giuravano che si sarebbero attenute alla decisione che sarebbe stata presa dai due giudici nel giro di ventiquattro ore ${ }^{37}$. Poi i due uomini - le donne non erano considerate soggetti idonei per una cerimonia così pubblica - si trattenevano per la disciplina, o mortificazione, che seguiva. A giudicare dalle relazioni i risultati della cerimonia potevano essere veramente impressionanti. Basterà un esempio. Nel 1668 a Soleto, nell'area grecofona chiamata la Grecìa, i missionari riuscirono a far rappacificare due opposti capi di una violenta faida e i loro rispettivi seguaci, dopo che un tentativo dei missionari durante la missione precedente era fallito. In questo caso singolare il normale trionfalismo delle relazioni è brevemente contraddetto dalle descrizioni delle reali difficoltà che incontrarono i Gesuiti nel missionare a Soleto. In un primo momento i missionari rifiutarono persino di accettare le richieste dell'arcivescovo di fare un'altra missione lì, dicendo che avevano "alijs civitatibus evangelizare» $\mathrm{e}$ chiamando in causa l'ostinazione e l'orgoglio di alcuni abitanti, la "greca fede» di altri (il rito ortodosso era ancora prevalente in alcuni paesi della

${ }^{35}$ Scipione Paolucci, Missioni de Padri della Compagnia di Giesù nel Regno di Napoli, Napoli, 1651, p. 127.

${ }^{36}$ Anna Maria Corbo, Relazione descrittiva degli archivi notarili romani dei secoli XIV-XV nell'Archivio di Stato e nell'Archivio Capitolino, in P. Brezzi e E. Lee (a cura di), Sources of social history : private acts of the late Middle Ages, Toronto, 1984, p. 4967, a p. 61-2. Ringrazio il Prof. Lee per questa segnalazione.

${ }^{37}$ Paolucci, Missioni, cit., p. 138. 
Grecìa), "la superbia di chi essendo stato il primo ad offendere non voleva ne meno dire una parola d'humiliatione», e «il pericolo di patir qualche danno per l'odio che due di una fattione haveano concepito contro li $\mathrm{Pa}$ dri». (Un pericolo vero, dato che la faida aveva già causato la morte di nove persone.) Ma alla fine decisero di sfidare il paese ancora una volta. L'ammissione da parte dei Gesuiti del loro iniziale fallimento e la riluttanza a continuare serviva solo a drammatizzare e mettere in risalto il risultato finale : la riconciliazione delle due fazioni in guerra e la pacificazione del paese.

Volle il Padre per stringergli meglio in santa Pace farli schiavi della Beata Vergine con una fune al collo, e tutti si ligarono con molta tenerezza d'affetto e lacrime. Così ligati calarono in chiesa ove l'altro Padre [missionario] data già la meditazione alle donne, le tratteneva col santo Rosario per impetrar dalla Vergine con S. Anna la pace. Subito che le donne li viddero così ligati diedero in pianti di giubilo e si pacificarono tutte le Mogli et altre parenti tra di loro. Corse in un momento la fama per la Terra e come se fosse accaduto un Terremoto tutti lasciarono ipso facto le case, i figli, e colle mani alzate in cielo benedicevano Iddio, la missione, i Padri e correvano alla Chiesa, le fanciulle ancora baciavano la terra dicendo più e più volte nella loro greca favella $l u$, loghimeno o theos, cioè benedetto Iddio $^{38}$.

Lo stile della prediche gesuitiche era particolarmente intenso. Questi "sacri oratori" cercavano di indurre i cuori e le menti dei loro ascoltatori alla confessione e al pentimento durante la breve durata della missione. Per raggiungere l'effetto desiderato, facevano uso frequente di materiale scenico : teschi, fruste, catene e torce (le ultime usate per ustionare la pelle e riprodurre i tormenti dell'inferno davanti ai fedeli). Alcuni oratori sacri erano esperti specializzati e avevano «per loro particolare mestiero l'andare in giro per tutto il Regno [di Napoli], facendo le missioni ${ }^{39}$. Uno di questi «divi» della missione era Onofrio Saraco (1605-50), che nonostante fosse affetto da una forte balbuzie, realizzò molte conversioni grazie alle sue prediche ferverose, come ci informa lo storico gesuita Paolucci ${ }^{40}$. Saraco faceva uso della tecnica seguente. Al suo arrivo in un paese, si informava segretamente sui nomi di alcuni notabili del paese morti di recente. Prima di cominciare una predica chiamava queste persone una per una. Quando gli veniva detto che erano morte di recente, si lanciava immediatamente nella sua predica - sulla morte e sulle pene dell'Inferno - «non senza un

${ }^{38}$ ARSI, Neap., 76 I, x, f. 53r-v.

${ }^{39}$ ARSI, Neap., 75, viii, f. 53.

${ }^{40}$ Paolucci, Missioni, cit., p. 278. La sezione riservata del Catalogo (catalogus secretus) per il 1649 descrive Saraco come un uomo di «boni ingenij, iudicij, ac prudentice ... aptus ad docendum rhetoricam \& ad missiones". ARSI, Neap., 83, f. 167. 
sensibile horrore di chiunque assisteavi; e come in cuori già mezzi guadagnati da quelli pietosissimi sentimenti, era non men facile che grande l'impressione che vi faceano le sue parole ${ }^{41}$ ».

La fondazione delle congregazioni mariane aveva come scopo quello di produrre un cambiamento permanente, o perlomeno di prolungare l'effetto della missione. Questo espediente fu impiegato specialmente dai Gesuiti. Secondo una relazione missionaria, «la fondatione delle congregationi in ciascheduna terra, che è il maggior bene che [le missioni] possino mai havere, perchè ivi con la frequenza de' Sacramenti, esortationi, e dscipline si procura mantenere tutto il bene fatto nella missione ${ }^{42}$ ». La rete di congregazioni, oratori e conservatori (per le ragazze) gesuitici si espanse rapidamente dal 1590 in poi attraverso l'attività missionaria, al punto da diventare una caratteristica e un segno relativamente permanente del passaggio dei Gesuiti attraverso le diverse aree del Regno, dagli importanti centri urbani alle più piccole comunità rurali ${ }^{43}$.

Le associazioni venivano formate per far sì che lo spirito missionario rimanesse più a lungo nella comunità, e perchè potessero condizionare la vita religiosa in un modo che i missionari da soli non potevano fare. Qualche volta i membri delle congregazioni accompagnavano i missionari gesuiti nelle loro missioni, partecipando alle processioni, cantando litanie alla Vergine, ascoltando le prediche, confessandosi, facendo atti di penitenza e mortificazione, preparandosi per la Comunione e facendo elemosine : in poche parole, mostrando quale era il modo migliore di seguire una missione e di trarne beneficio ${ }^{44}$. Inoltre, le congregazioni consentivano ai laici di esercitare ruoli importanti, tra cui quello di rappresentare un esempio cristiano per la comunità attraverso le opere di devozione e di carità, di insegnare il catechesimo e di incoraggiare una fede "corretta" negli altri ${ }^{45}$. Le pratiche adottate - la preghiera mattutina, l'esame di coscienza serale, la confessione bimestrale, la frequente comunione e i ritiri spirituali - finirono con l'essere accettate dalla gente come modelli devozionali (anche se non erano sempre messe in practica).

Queste confraternite non erano solo per i laici. Infatti, la fondazione di congregazioni speciali per il clero locale fu un'iniziativa importante, in quanto era solo il clero che poteva far sì che lo stimolo dato dalla missione

${ }^{41}$ Paolucci, Missioni, cit., p. 281.

${ }^{42}$ ARSI, Neap., 76 I, iv, f. 28.

${ }^{43}$ Cfr. Rosa, Strategia, cit., 165.

${ }^{44}$ Louis Châtellier, L'Europe des dévots , Parigi, 1987, p. 36.

${ }_{45}^{45}$ Pasquale Lopez, Le confraternite laicali in Italia e la Riforma Cattolica, in Rivista di studi salernitani, II, 1969, p. 153-238, a p. 183. 
non si dissolvesse subito dopo. Per tutta la prima età moderna il clero locale dell'Italia meridionale continuò a essere tristemente inadeguato, nonstante le riforme proposte dal Concilio di Trento. In Terra d'Otranto questo era dovuto soprattutto alla mancanza di seminari stabili - la maggior parte delle diocesi non ebbero affatto seminari fino alla metà del ' 700 - e all'organizzazione del clero in chiese "ricettizie», la cui struttura chiusa e collegiale rendeva il clero restio alle riforme ${ }^{46}$. L'insistenza dei Gesuiti sulla comunione frequente e su una conoscenza del catechismo, per quanto elementare, capovolse regole e tradizioni di vecchia data ${ }^{47}$. Comunque si resero conto che il successo o il fallimento delle loro riforme e devozioni dipendeva da un clero ben preparato e ben disposto. Dove, quindi, i missionari gesuiti trovavano un clero locale non istruito e incapace persino di celebrare la messa in modo adeguato, la creazione di una congregazione era un modo per migliorare la situazione ${ }^{48}$. Oppure, dove il clero non adempiva al suo dovere di dire le messe per i defunti, gli veniva fatta una predica con esercizi spirituali sull'argomento (per un risultato immediato), e veniva istituita per loro una congregazione "della buona morte" (per un risultato a lungo termine) $)^{49}$. Tali tentativi non erano sempre ben accolti, come vedremo nel prossimo paragrafo.

Come si comportano a riguardo le altre congregazioni missionarie? Per rimanere nell'ambito delle associazioni pie, c'è da dire che i Lazzaristi cercarono di stabilire una "compagnia della carità» in ogni parrocchia in cui facevano missione, con un intento simile a quello delle congregazioni mariane dei Gesuiti. Comunque, non tutte le congregazioni missionarie erano favorevoli alla creazione di associazioni pie per i laici. I missionari delle Apostoliche missioni, istituite a Napoli nel 1646, riservavano le loro congregazioni - dedicate a "casi di coscienza" - solo per il clero. Questa dedicazione costituisce un ulteriore esempio dell'importanza attribuita al confessionale come luogo di consolidamento della conversione religiosa. Inoltre, l'esperienza fatta dai Missionari apostolici insegnò loro che le associazioni laiche, "oltre che alienano i Parrocchiani dalle Chiese matrici, diventano finalmente un semenzaio di litigi ${ }^{50} »$. Nel momento in cui questo

${ }^{46}$ Parlo della "condizione del clero» e la riforma tridentina nel secondo capitolo di From bishop to witch. The system of the sacred in early modern Terra d'Otranto, Manchester, 1992.

${ }^{47}$ Prosperi, Missionario, cit., p. 205.

${ }^{48}$ Questo metodo fu adoperato durante una missione del 1650; cfr. ARSI, Neap., 75, ii, f. 21v.

${ }^{49}$ ARSI, Neap., 76 I, xi, f. 68-9.

${ }^{50}$ Regole particolari per le sante missioni di fuori della Congregazione delle Apostoliche Missione, Napoli, 1768, p. 36 e 45-6; cit. in Marcello Semeraro, Le apostoliche 
veniva scritto, nelle loro regole per fare missioni (del 1768), le confraternite laiche venivano guardate, in misura sempre crescente, come un impedimento alla creazione di una struttura parrocchiale forte e florida. Ciò che una volta era visto come una soluzione al problema, ora lo si credeva una sua perpetuazione. La parrocchia stessa, e la preparazione del clero parrocchiale, assunsero un'importanza sempre maggiore per le missioni del tardo Settecento, e ne avrebbero costituito l'interesse predominante durante il Novecento.

Anche se tutte le congregazioni missionarie in gran parte rifiutarono la processione penitenziale dei Gesuiti, per ragioni che esploreremo nel paragrafo finale, preparavano con cura la messa in scena degli arbitrati pubblici tra parti in disputa, che erano così importanti per i Gesuiti. Comunque, questo non significa necessariamente che le altre congregazioni accettassero anche il tipo di predica che accompagnava tali occasioni. La varie congregazioni reagirono in maniera alquanto differente ai modi di predicare usati dai Gesuiti. I predicatori lazzaristi, per esempio, adottarono uno stile molto diverso di predicazione, basato sulla semplicità e sulla schiettezza, come abbiamo detto sopra. Anche se gli argomenti che sceglievano per le loro prediche erano simili sia a quelli dei Gesuiti che a quelli dei Redentoristi, i missionari lazzaristi cercavano di ottenere la conversione mettendo in rilievo la natura intrinseca dei loro temi, piuttosto che usare i mezzi esteriori e teatrali utilizzati dagli altri gruppi.

Il metodo gesuitico fu, in molti modi, perpetuato dai Redentoristi. Come abbiamo già notato, i Gesuiti e i Redentoristi condividevano lo stesso stile emotivo, gli ultimi prendendo molto in prestito dai primi. Una tecnica dei Redentoristi per richiamare le immagini degli eterni tormenti dell'inferno era quella di rivolgersi a un "paesano deceduto" perché raccontasse dei tormenti dell'inferno all'assemblea dei fedeli. Nella chiesa buia il predicatore, sul palco, accendeva una torcia sotto un teschio che teneva in mano e con il quale conduceva un dialogo, penendogli domande, quali (a mo' di esempio) "che si fa nell'altra vita?", e rispondendo con un tono di voce differente ${ }^{51}$. Un'altra predica, ancora più teatrale, era chiamata la "predica dei veli» e aveva come soggetto il Giudizio universale. Dopo una predica in cui si invitavano tutti i peccatori a pentirsi prima che fosse trop-

missioni. La congregazione dei 'Padri Salesiani'o 'Preti Pietosi' nel Sette-Ottocento Leccese, Roma, 1980, p. 36.

${ }^{51}$ Gagliardi, Direttorio, cit., f. 154. Una predica simile del Redentorista Ludovico Altarelli è citata da Gabriele De Rosa, Vescovi, popolo e magia nel Sud, Napoli, 1983, 215. 
po tardi, il predicatore doveva fare il gesto di sollevare il crocifisso per dare la benedizione ma poi doveva dire :

"Come voglio benedirli", mi ha detto Gesù Cristo, «se un giorno dovrò maledirli? E perciò no, non posso benedirli stasera», mi ha detto Gesù Cristo. Dunque, peccatori, non ci è benedizione stasera, anzi Gesù Cristo ti ha voltato le spalle.

A questo punto il predicatore doveva girare il crocifisso in modo da far "vedere le spalle» alla gente e doveva coprirlo con uno dei veli. Ma questo era solo l'inizio. (In base a precise istruzioni, il predicatore doveva togliere la corona dalla statua della Madonna prima della predica e procurarsi degli spilli, "perchè l'ago non è buono».) Poi invitava i peccatori a fare ricorso alla Vergine Maria :

Ma sapete che dice Maria? ... "Io non sono più Regina di Misericordia, e perciò da ora mi levo la corona ». (La quale [corona] girata sul palco si depone sulla sedia, e poi si ritorna colle torce a Maria, e si dirà :) E come allora non sarai più nostra Regina? "Che Regina», dice Maria, "non vi sarò neppure Madre, e per non vedervi perduti mi ricuoprò la faccia». (E qui coll'altro velo si ricuoprirà la Madonna. In tanto si alzerà il crocifisso ricuoperto, la Madonna velata, e si dirà :) Non ci è benedizione. (Ed in così dicendo, [il predicatore] smorzerà le torce, le candele innanzi la Madonna e le altre lampade) ${ }^{52}$.

Tramite le loro ferventi prediche e la diffusione di devozioni emotive come lo strascino - appropriatamente praticato dopo una predica sulla bestemmia - i Redentoristi rifiutavano la fede austera che veniva proposta in molte sfere della Chiesa durante la seconda metà del Settecento, e parlavano direttamente alla gente che dovevano evangelizzare. La penultima sera di una missione solevano "piantare" cinque croci del Calvario fuori dal paese e distribuire piccole croci benedette, che portavano indulgenze, da "mettersi in casa", tutto in memoria della missione ${ }^{53}$. Come ricordo tangibile della missione i Redentoristi distribuivano anche rosari benedetti e i cosiddetti «abitini di Maria». Questi ultimi erano piccole borsette di stoffa, con stampata sopra l'immagine della Madonna o di un santo, che venivano indossati come scapolari intorno al collo o cuciti ai vestiti per proteggere chi li portava, ed era diffusa la credenza popolare che avessero poteri taumaturgici.

Quale fu l'impatto di questa attività missionaria in Terra d'Otranto? In che misura corrispose alle aspettative degli stessi missionari? Quello che i missionari gesuiti ritenevano un risultato di successo per una missione vie-

52 Gagliardi, Direttorio, cit., f. 173-4.

${ }^{53}$ Ibidem, f. 267-8. 
ne indicato alla fine della relazione di una missione del 1639, citata all'inizio del paragrafo precedente. Essa sottolineava le confessioni e le conversioni ottenute e il riconoscimento dato alla missione :

Si sono rifatte confessioni di molti anni malamente fatte, et anco di tutta la vita. Si sono fatte molte restitutioni di diverse quantità di denari, come si è saputo dalle persone alle quali sono stati resituiti, che sono venuti a ringratiarci. Molti si sono confessati, non si essendo confessati per spazio di 20, e 25 anni... é parsa Lecce un'altra Nineve convertita; adesso pare che conosca la Compagnia : non si può caminare per le strade, che non ci benedichino et honorino tutti ${ }^{54}$.

I «successsi " delle missioni gesuite, ciò che potremmo definire il loro impatto, sono relativamente facili da documentare per quanto riguarda il breve termine. Prendiamo di nuovo l'esempio della confessione. Le relazioni e le osservazioni riportate nelle lettere annuali sono abbastanza specifiche a questo riguardo. Un topos frequente è la descrizione dell'elevato numero di fedeli impazienti di confessarsi durante la missione : per esempio, le folle che si radunanvano fuori della chiesa la notte prima per potersi confessare a prima mattina. Una variante era che la folla era così numerosa e al di sopra della aspettative che dovevano essere chiamati altri confessori. Ma questi sono più che semplici motivi letterari. La paura di dannazione imminente che scaturiva dalla missione, portava la gente al confessionale. Conduceva inoltre a conversioni e pentimenti pubblici e alla partecipazione alle processioni penitenziali.

Le relazioni missionarie abbondano di dettagli che riguardano gli effetti immediati delle processioni. Una processione penitenziale «diede tanta divotione" che una persona che assisteva allo spettacolo "si levò subito per andare a confessarsi d'una risolutione che teneva d'uccidere sua moglie, et un huomo dal quale era stato dishonorato, perdonando all'uno, e l'altro, pacificandosi anche al nemico " ${ }^{55}$. Tale era l'ansia creata e il desiderio di partecipare alla "disciplina" che la gente si batteva con qualunque oggetto a disposizione. Nelle zone rurali gli uomini si battevano con attrezzi agricoli, come le catene usate per legare insieme gli zoccoli dei cavalli, le cavezze per i muli «et ogni altro instrumento di ferro poteano havere" ${ }^{56}$. L'intensità di queste mortificazioni era certamente autentica, tanto che riesce a penetrare anche la densa retorica della relazioni. Una dettagliata descrizione di una processione penitenziale del 1630 racconta che "qui ferreis catenulis adeo se verberarunt, $u$ t non ruberent tantum sanguinem, sed dilace-

${ }^{54}$ ARSI, Neap., 73, xliv, f. 455.

55 Ibidem, f. 454.

${ }^{56}$ ARSI, Neap., 74, xvi, f. 215. 
ratis etiam carnibus detectis ossibus albicarent ${ }^{57}$ ". La disciplina spesso aveva luogo di sera, dopo una processione, all'interno della chiesa e le donne venivano mandate a casa. Nonostante fossero incoraggiate a pregare e a compiere atti di contrizione a casa, le donne qualche volta si trattenevano fuori della chiesa, «e mentre quelli si battono, queste gridano con lacrime e sospiri ad alta voce misericordia a Dio $^{58}$ ".

Un fervore collettivo sembra caratterizzare varie fasi della missione, dalle processioni penitenziali alla messa in scena delle pacificazioni e dei pentimenti pubblici. Questa febbre di emozione, tipica dei periodi di grande tensione, era molto contaggiosa all'interno della comunità : ad un pentimento spesso ne seguiva un altro. Nel 1655 in una missione gesuitica a Torre Paduli alcuni adulteri confessarono i loro peccati e insieme fecero una processione penitenziale, che culminò con lo strascino dalle porte della chiesa all'altare. I concittadini gridavano «una omnium voce» che il male era stato cacciato fuori dal paese ${ }^{59}$. Le descrizioni di tipo numerico riflettono questo fervore : quella tipica è il topos di dover predicare o celebrare la messa all'esterno o in un campo fuori del paese perché la chiesa locale non poteva contenere la folla che si radunava. I contadini sacrificavano ore del loro lavoro, spesso in campi lontani, per ascoltare le prediche, e suore malate spesso si privavano delle medicine e delle visite mediche durante gli esercizi spirituali che si tenevano nei conventi ${ }^{60}$.

Quando, sul palco, il predicatore si flagellava o si bruciava, alcuni dei fedeli prendevano parte a questa mortificazione schiaffeggiandosi o battendosi con pietre o con corde, contribuendo ad accrescere l'atmosfera di tensione e di ansietà. Non c'è da meravigliarsi che questo suscitasse il pianto o il terrore nella gente. Il rapporto che si stabiliva tra il predicatore e i fedeli in queste sacre rappresentazioni si sviluppava ulteriormente quando venivano bruciate le carte da gioco, i nastri e altri ornamenti, e i libri osceni e proibiti. Le relazioni riportano casi in cui alcuni componenti del pubblico prendevano in mano il crocifisso e con questo sostenevano un dialogo, nello stile dei predicatori ${ }^{6}$. Ma più spesso le relazioni raccontano reazioni di terribile paura e disperazione. Nel corso di una predica

${ }^{57}$ ARSI, Neap., 73, xxix, f. 258r.

${ }^{58}$ Ibidem, xii, f. 113v.

${ }^{59}$ ARSI, Neap., 75, xi, f. 86v.-87r. L'intero resoconto viene presentato nei termini di una conquista militare. Infatti, in tutte le relazioni è presente una terminologia militare - comuni sono le "excursionculae" nelle campagne - accompagnata da una terminologia agriculturale ("curare la vigna», "fare una buona raccolta»).

${ }^{60}$ ARSI, Neap., 75, vii, f. 41v., per un esempio.

${ }^{61}$ ARSI, Neap., 76 I, x, f. 54v. 
sulla morte, tre donne "perillustre" - riporta la relazione del 1734 - si precipitarono sul palco e si gettarono in ginocchio di fronte alla folla, implorando misericordia e chiedendo preghiere. E più tardi durante la stessa predica un uomo si precipitò verso il predicatore e gli strappò la frusta dalle mani, si inginocchiò davanti al crocifisso e si frustò, piangendo intensamente ${ }^{62}$.

I missionari cercavano di trarre vantaggio da questa ondata di devozione e dallo sfogo di emozione - un misto di fervore e di paura - per ottenere conversioni. Le tecniche istrioniche usate nel predicare erano tali da provocare le critiche del prete (di istruzione gesuitica) e bibliotecarioarchivista Ludovico Antonio Muratori (1672-1750). «Ho conosciuto io alcuni [predicatori] - scrisse - i quali si abbassavano, si contorcevano, e dimenavano le braccia a tutto potere, saltellando di qua e di là sul pulpito, stendevano stoccate a tutte le parti... Altri con istirature curiose di corpo imitavano un peccator disperato, un martire sul eculeo, un'anima dannata $^{63}{ }^{3}$. Muratori criticò lo stile missionario di predicare che si concentrava sulle pene dell'inferno e sulla severità del giudizio divino, al quale nessuno poteva sfuggire. Lui scrisse : "Ho conosciuto Femmine, che in occasion di una strepitosa sacra Missione son cadute in insania, e s'è poi durata fatica a rimetterle in sesto ${ }^{64}$ ). Le vivide immagini presentate nel corso di una predica - e una missione che durava otto giorni significava altrettante prediche, per non parlare delle omelie più brevi e dei fervorini - avevano il loro effetto sulla gente del luogo. Nel 1651, durante una missione gesuitica, un prete locale sognò Cristo sulla croce, che grondava di sangue dalle ferite, che lo rimproverava per i suoi modi lincenziosi (il prete aveva una concubina). Turbato, il prete si svegliò e - come dopo raccontò a un missionario - gli apparve S. Francesco Saverio che gli mostrò scene dell'inferno, in cui appariva anche un drago che sputava fuoco. Quando il prete cadde a terra per il terrore, il santo fece svanire l'apparizione e le sue parole gentili portarono il prete al pentimento ${ }^{65}$. Le relazioni e le lettere annue sono piene di questo tipo di sogno/visione, che spesso continene scene dell'inferno o di una discesa agli inferi, il tutto raccontato nei minimi particolari ai missionari. Spesso le prediche sembrano produrre una specie di delirio, come nello strano caso seguente dove una donna vede il predicatore come Cristo,

con una chioma bionda sino alle spalle tre volte, e una con la corona di

${ }^{62}$ ARSI, Neap., 76 II, lxxiii, f. 542.

${ }^{63}$ L. A. Muratori, Dei pregi della eloquenza popolare, 2a ed., Venezia, 1750, p. 30.

${ }^{64}$ L. A. Muratori, Della forza della fantasia umana, $2^{\text {a }}$ ed., Venezia, 1753, p. 119.

${ }^{65}$ ARSI, Neap., 75, iv, f. 30. 
spine in testa, la fronte, et il volto spruzzato di sangue... Vedendo dunque il Padre in quella guisa si battea il petto con tute due le mani a pugni serrati a vista di altre donne, che udivano la predica; dicendo fra se, che non era degna di veder Christo in quel modo. Subito finita la predica se ne andò a trovare il predicatore, et ancorchè ci fossero huomini, e donne si pose dal principio della porta a caminare per due stanze con la lingua per terra sino ad arrivare dove stava il P. predicatore, e gli disse, "tu sei Christo per me, come l'ho visto nella faccia tua", raccontandole il tutto, come ancora de verbo ad verbum dopo lo ratificò in confessione al P. Auriano, che l'havea confessata anco prima del successo ${ }^{66}$.

Questa emotività raggiungeva l'apice nel modo in cui i missionari stessi venivano trattati dalla popolazione locale. Spesso erano ritenuti «santi vivi", con tutti i poteri - e l'ambivalenza - che questa sacralità comporta$\mathrm{va}^{67}$. Questa è una caratteristica presente anche nelle missioni del Nuovo Mondo. Lì la desacralizzazione delle credenze e pratiche indigene era accompagnata da un afflusso del sacro nella forma della sacralizzazione dei missionari stessi. A Nuova Granada (Colombia), per esempio, gli Indios consideravano i Gesuiti degli sciamani. Inoltre, «idolatravano» gli oggetti che i missionari introdussero, come il rosario ${ }^{68}$. Nell'Italia meridionale (e senza dubbio anche altrove) troviamo lo stesso atteggiamento popolare verso il potere del rosario. Qui, l'importanza che i missionari attribuirono al rosario - la "scala del paradiso" secondo una predica redentorista ${ }^{69}$ - ebbe un impatto più duraturo, anche se la cultura popolare fu in grado di sviluppare la propria interpretazione riguardo alle sue virtù. Da strumento di meditazione, il rosario acquistò gli attributi di un articolo devozionale con tutto il potere di una reliquia. I Missionari apostolici contribuirono a questo cambiamento dimostrando l'importanza del rosario con le «narrazioni di miracoli" : episodi che illustravano la protezione miracolosa offerta dalla Madonna del rosario ai suoi devoti. Comunque, allo stesso tempo, l'efficacia della preghiera meccanica e ripetuta propria del rosario poteva subire una distorsione da parte della cultura popolare : come avvenne con il rosario recitato per le anime degli «impisi giustiziati». Secondo una denuncia del 1742 al tribunale vescovile di Oria, questo "rosario si doveva

${ }^{66}$ ARSI, Neap., 74, xvi, f. 216.

${ }^{67}$ Per studi recenti sulle "sante vive», cfr. le note in David Gentilcore, The Church, the devil and the healing activities of living saints after the Council of Trent, in O. P. Grell e A. Cunningham (a cura di), Medicine and the Reformation, Londra, 1993, 134-55 (ora in : Gentilcore, Healers and healing in early modern Italy, Manchester 1998, capitolo 6).

${ }^{68}$ Serge Gruzinski, Christianisation ou occidentalisation? Les sources romaines d'une anthropologie historique, in MEFRIM, 101, 1989, p. 733-50, a p. 744 e 748.

${ }^{69}$ Gagliardi, Direttorio, cit., f. 22. 
recitare colle mani dietro, all'oscuro, e senza voltarsi indietro, perché voltandosi indietro chi lo recitava, vedeva le teste degl'Impisi colla lingua di fuori». La donna che descriveva questa pratica fece l'errore di girarsi e così vide la scena infernale ${ }^{70}$.

Allo stesso modo l'impatto del messaggio missionario che insisteva sulla necessità di una confessione completa e sincera, non sempre era quello voluto dai missionari. L'importanza che attribuivano al confessionale poteva assumere diversi significati che esprimevano i bisogni immediati della cultura locale. Per esempio, S. Francesco de Geronimo era stato un confessore instancabile durante le sue missioni. Dopo la sua morte, però, non fu l'aumento dell'uso della confessione tra $\mathrm{i}$ fedeli che colpì maggiormente $\mathrm{i}$ testimoni che deponevano al processo per la sua beatificazione. Fu invece il fatto che i malati si sedevano (o venivano fatti sedere) per un po' di tempo nel confessionale dove il santo era solito ascoltare le confessioni, nella speranza di ottenere una guarigione miracolosa. Inoltre, schegge del confessionale venivano prese come reliquie curative ${ }^{71}$.

Ma torniamo alla diffusa sacralizzazione degli stessi missionari. Secondo la testimonianza di Scipione Paolucci la gente aveva paura di lasciare il paese durante le missioni perchè temeva di venire esclusa dal Paradiso; una credenza, questa, che i Gesuiti non cercarono in nessun modo di contraddire $^{72}$. Paolucci continua : "In occasione di terremoti, mentre si predicava s'affollò tutta la gente intorno a' Padri missionanti, per morire, diceano essi, vicino a' Padri santi, ò in virtù de' lor meriti liberarsi da mor$\mathrm{te}^{73}$ ». Questo misto di paura, devozione e una credenza nel potere apotropaico dei missionari accompagnavano i Gesuiti quando lasciavano un paese per evangelizzarne un altro. Venivano seguiti «dal clero, huomini, e donne, da tutto il popolo ... cantando hinni, e cantici spirituali». Uno dopo l'altro, alcuni fra i seguaci si avvicinavano ai missionari per «bacciarli le mani, i bordoni, la muzzetta, il crocefisso [che] portavano in petto, toccandole adosso le corone», per impregnare i rosari del loro sacro potere ${ }^{74}$. In un'occasione questa "pia credulità indusse la gente a tagliare pezzi degli abiti indossati dai missionari, "conservati non meno per riverenza degli altrui meriti, che per rimedio de' proprij mali ${ }^{75}$ ». Eppure l'equiparazione dei

${ }^{70}$ Archivio diocesano, Oria, Magia, II, "Dorotea Rossi denuncia diverse donne per pratiche magiche», 24.7.1742.

${ }^{7}$ ASV, Con. Riti, no. 2022, f. 339r-341v.

72 Paolucci, Missioni, cit., 75.

${ }^{73}$ Ibidem, 273.

${ }^{74}$ ARSI, Neap., 74, xvi, f. 215v.

${ }^{75}$ Paolucci, Missioni, op. cit., p. 273. 
missionari ai santi non era semplicemente una questione di credenza popolare che scaturiva dalla ritenuta vicinanza dei missionari a fonti di potere sacro. La totale dedizione dei missionari, il loro ascetismo, la loro abnegazione e le loro opere miracolose corrispondevano ai modelli ortodossi di santità del periodo. La santità dei missionari, "come i loro sermoni, pur avendo qualcosa di teatrale, era reale», secondo Jean Delumeau. "Ascoltatori e penitenti avevano la sensazione di trovarsi davanti a sacerdoti eccezionali che sacrificavano alla loro missione apostolica benessere e salu$\mathrm{te}^{76} \%$. Comunque questo sacro potere aveva anche le sue connotazioni negative. Questa ambivalenza è tipica del concetto popolare del sacro, che includeva sia il divino che il diabolico, il bene e il male, la guarigione e la malattia. Perciò durante una missione a Matera nel 1687 i missionari si trovarono di fronte a una forte opposizione alla loro presenza da parte della gente del luogo. Questo era dovuto al fatto che alla loro ultima missione, dieci anni prima, aveva fatto seguito un anno di siccità e di carestia, che la gente del paese aveva attribuito alla missione ${ }^{77}$.

L'accoglienza che i missionari ricevevano dalla popolazione locale poteva condizionare in misura rilevante l'impatto, a breve e a lungo termine, di una missione. Nei primi anni, per esempio, la novità delle missioni aveva spesso come risultato resistenza e ostilità a livello locale. Nel 1618, in occasione della loro prima visita in un paese, si sparse la voce che i Gesuiti fossero ufficiali del governo giunti per applicare una nuova gabella di venti grane a famiglia ${ }^{78}$. In seguito, naturalmente, finirono con l'essere trattati come santi dalla popolazione locale, secondo questo topos delle relazioni. Perciò bisogna affrontare le relazioni con cura, e con una buona dose di scetticismo : furono scritte in modo tale da far apparire i missionari e la loro attività in una luce positiva se non addirittura eroica. Una missione che durava solo una settimana non poteva sperare di eliminare pratiche che facevano parte della coscienza popolare da secoli. Eppure la seguente affermazione è tipica :

Per l'inavertenza de confessori le superstitioni in due terre erano infinite, si commettevano da tutti senza scrupoli, e per ogni negotio ò di matrimonio, ò di peccato, ò di medicamento, ò di lite, si ricorreva à superstitioni, magarie,

${ }^{76}$ Delumeau, Missioni, op. cit., p. 223.

${ }^{77}$ ARSI, Neap., 76 I, xxxiii, f. 185. Ad indicare questa ambivalenza ci sono i casi dove la presenza dei missionari venne richiesta durante calamità naturali come carestie o invasioni di bruchi, dove $\mathrm{i}$ "rimedi ecclesiastici" del clero locale - benedizioni, esorcismi, processioni con reliquie - erano falliti.

${ }^{78}$ ARSI, Neap., 73, xii, f. 114. 
et altre cose diaboliche, con queste missioni si è rimediato notabilmente con estremo gusto de Prelati aiutati, e de Popoli illuminati ${ }^{79}$.

Ovviamente tali successi, nella migliore delle ipotesi, erano temporanei. Troviamo la stessa situazione nella lotta dei Gesuiti contro il gioco d'azzardo, le bestemmie e la circolazione di libri proibiti. Altre fonti storiche - dalle cronache locali agli atti giudiziari ${ }^{80}$ - risulterebbero utili in quanto consentirebbero di collocare le affermazioni delle relazioni missionarie nella giusta prospettiva (o, se preferiamo, contesto), ma mettere insieme le varie indicazioni sparse di qua e di là richiederebbe un lavoro da certosino, e molto dipenderebbe dalla fortuna di trovare documenti rilevanti negli archivi. Quale potè essere, per esempio, l'impatto prodotto da un rogo di libri che si svolse a Francavilla nel 1678, quando un praticante di magia naturale del luogo, un certo Nicola Salinaro, poteva nascondere i suoi libri di magia, fino alla partenza dei missionari, senza sentirsi in colpa? In questo processo episcopale per la pratica di magia colta la presenza dei missionari gesuiti appare come un motivo ricorrente. Furono le loro prediche che persuasero una delle testimoni a confessare la sua conoscenza dell'imputato, Salinaro, all'arciprete del paese e a deporre contro di lui ${ }^{81}$. Un altro testimone cominciò a chiedersi se i rituali di guarigione impiegati dall'imputato non fossero in realtà magici e diabolici. Per rassicurarlo, Salinaro gli disse di aver bruciato tutti i suoi libri di magia durante la missione $^{82}$. Tuttavia, mentre i Gesuiti potevano sforzarsi di combattere le forme popolari di magia con le loro devozioni ortodosse, non avevano niente da offrire al posto della magia dotta, praticata normalmente da membri dell'élite colta, sia laica che clericale. Perciò il mago imputato in questo processo reagì ai missionari non bruciando i suoi libri come proclamava di aver fatto, ma nascondendoli, come fece più tardi prima di essere arrestato

${ }^{79}$ ARSI, Neap., 76 I, vii, f. 40r-40v.

${ }^{80}$ Sotto la categoria di atti giudiziari si potrebbero includere anche i documenti dei processi di beatificazione e canonizzazione. Le deposizioni dei testimoni possono rivelare molto, e ho consultato i documenti che riguardano S. Francesco de Geronimo, originario di Terra d'Otranto, che fece molte missioni in tutto il Regno di Napoli (cfr. $\mathrm{n} .8 \mathrm{e} \mathrm{n.} \mathrm{71).} \mathrm{Tuttavia,} \mathrm{data} \mathrm{la} \mathrm{natura} \mathrm{della} \mathrm{fonte,} \mathrm{le} \mathrm{testimonianze} \mathrm{tendono} \mathrm{a}$ mettere in risalto i doni e i successi straordinari di de Geronimo, che lo qualificano per una eventuale canonizzazione. I testimoni che assistettero alle sue missioni insistono sulla sua santità fino al punto che diventa difficile immaginare in che misura fossero influenzati da missioni ordinarie, fatte da missionari ordinari.

${ }^{81}$ Archivio diocesano, Oria, Magia, III, deposizione di Donatantonia de Milato, "Contro Nicodemo Salinaro per sortilegi», Francavilla 1678, f. 52r.-53r.

${ }^{82}$ Antonia Quaranta, ibidem, f. 86v.-87v. 
e incarcerato ${ }^{83}$. Chiaramente un unico esempio di questo tipo è di limitata utilità; ma, se non altro, ci mette in guardia quando leggiamo documenti scritti non per il diletto degli storici ma per la maggior gloria della Compagnia di Gesù.

Quando le relazioni fanno riferimento alle difficoltà incontrate dai missionari, servono solo a dare maggiore drammaticità ai loro trionfi finali. Malgrado questo scopo, tali occasioni sono importanti in quanto ci permettono di vedere le tensioni presenti e il tipo di opposizione che i missionari incontravano. Ci offrono anche l'opportunità di approfondire il «codice" delle relazioni, anche se in misura limitata, e di ricostruire quelle condizioni, quegli avvenimenti e quelle reazioni che si tacevano perchè mettevano in cattiva luce i missionari e il loro lavoro. A mo' di esempio, con riferimento all'opposizione alla missione di Matera del 1687, a cui si è fatto riferimento sopra (nel contesto dei poteri sacri dei missionari), la relazione riporta anche che molti abitanti del paese avevano fatto fervorose mortificazioni durante la missione precedente e che, una volta raffreddatosi il fervore, si erano pentiti di quello che avevano fatto e "per molto tempo chi per scherzo, e chi per altro fine se la rinfacciavano l'un l'altro, onde che ne succedevano varie rissa frà cittadini; e così impressionati in nessun modo volevano le missioni ${ }^{84}$ ".

Perciò il ricordo spiacevole di una precedente missione poteva avere come risultato un'opposizione verso le missioni successive. Nel 1699 un Gesuita scrisse che molte persone

si lagnavano con modi improprii della stracca data loro per le processioni continue, sì che avevano avuto occasione per bestemmiare, quando tornavano a casa ad un'ora e mezza di notte et al buio, e si lagnavano altresì della libertà nel parlare dal pulpito, con riferirne alcuni fatti, quali convenne interpretare in buona parte ${ }^{85}$.

Anche se, stando alle relazioni dei Gesuiti, questa opposizione veniva inevitabilmente vinta, si possono trovare tracce di una più diffusa opposizione verso il carattere "popolare» ed eccessivo della missione gesuitica a partire della seconda metà del Seicento. Questa opposizione crebbe nel corso del Settecento, quando la missione veniva sempre più associata alle campagne. Il modo istrionico di predicare e le macabre mortificazioni non

${ }^{83}$ Tommaso de Milato, ibidem, f. 166v.-168v.

${ }^{84}$ ARSI, Neap., 76 I, xxxiii, f. 185.

${ }^{85}$ ARSI, Rom., 136, relazione del P. Pier Maria Terusio a P. Bernardini, Provinciale di Roma, 16.6.1699, ff. 169-70; cit. da Giuseppe Orlandi, Missioni parrocchiali e drammatica popolare, in SHCSR, XXII, 1974, p. 313-48, a p. 341. 
erano più ritenute idonee alle città "colte ${ }^{86}$ ». Le élites provavano imbarazzo e criticavano tali pratiche. Già nel 1688 , nel paese di S. Pietro in Galatina, i missionari incontrarono l'ostilità di coloro che giudicavano la loro presenza come un'allusione ad una supposta inferiorità del paese, come se si insinuasse che fosse sperduto e arretrato, bisognoso di evangelizzazione. Nella relazione sono riportate le lamentele di un abitante : "Che pretendono questi Gesuiti da Noi, forse che non crediamo in Dio? ò pensano, che la Terra di S. Pietro la più celebre di questa Provincia sia qualche casalotto? " Attenendosi allo scopo e al tono generale delle relazioni, questa conclude dicendo che quella gente che si era lamentata della presenza dei missionari diventò alla fine la più penitente ${ }^{87}$.

In alcuni casi i Gesuiti incontravano l'ostilità del clero locale. La sola presenza di una missione in una comunità sembrava suggerire che il clero era venuto meno alle proprie responsabilità pastorali. Il gesuita Antonio Baldinucci (1665-1717), tuttavia, dava poco conto all'opposizione del clero locale, attribuendola al loro orgoglio :

La superbia ... travestita ben spesso d'abito ecclesiastico e religioso, tende più autorevoli ed insieme più pregiudicali le sue menzogne, [asserendo] che le missioni stanno bene tra gl'infedeli che non credono, o al più tra' bifolchi di campagna, indocili ed ignoranti, e non in terre culte e civili, e molto meno nelle città degne di gran rispetto, e dove non manca chi possa al pari de' missionarii e instruire gl'ignoranti, e correggere i traviati ${ }^{88}$.

Fu a causa delle critiche e dell'ostilità di queste élites che il gesuita Paolo Segneri junior (1673-1713), per esempio, preferì condurre la sua opera di evangelizzazione nelle campagne. Riteneva inoltre che le popolazioni rurali - mancando di una solida base pastorale - avevano maggiore bisogno delle missioni. La sua esperienza lo portò anche a preferire le missioni parrocchiali a quelle tradizionali di tipo centrale diffuse da suo zio, Segneri senior $^{89}$. Ma si trattava soltanto di una differenza di grado. In una lettera a Segneri jr. nell'estate del 1712, Muratori criticò alcuni eccessi come, per esempio, gli abiti indossati e gli strumenti utilizzati dai penitenti, la quasi nudità degli uomini durante le flagellazioni publiche e l'importanza data al

${ }^{86}$ Per esempio, le missioni urbane gesuitiche furono vietate a Milano nel 1767 , prima della soppressione della Compagnia, ma i Gesuiti poterono continuare a fare le loro missioni rurali. Cfr. Vismara Chiappa, Forme, cit., p. 827.

${ }^{87}$ ARSI, Neap., 76 I, f. 56v.

${ }^{88}$ ARSI, Opera nostrorum, 299, Antonio Baldinucci, "Avvertimenti utilissimi a chi desidera impiegarsi nelle missioni, cavati dall'esperienza di quei che le han fatte per molti anni», f. 5; cit. da Orlandi, Missioni parrocchiali, cit., p. 339.

${ }^{89}$ Giuseppe Orlandi, L.A. Muratori e le missioni di P. Segneri Jr., in SHCSR, XX, 1972 , p. $158-93$, a p. $172-4$. 
culto delle immagini. Suggerì che sarebbe stato necessario apportare solo pochi cambiamenti alla strategia missionaria, ma la risposta di Segneri fu un rifiuto categorico ${ }^{90}$. E questo veniva da un amico e ammiratore di Segneri jr, che avrebbe scritto la sua biografia sette anni dopo la sua morte ${ }^{\text {!! }}$ La critica era molto più seria quando veniva da persone al di fuori del giro dei Gesuiti, come il movimento quietista e i gruppi giansenisti. Questi ultimi criticavano non solo gli «eccessi» della missione gesuitica, ma anche le basi teologiche su cui ritenevano che si reggesse. Riferendosi alla pratica gesuitica dell' «accomodamento", i Giansenisti accusavano i Gesuiti di essere influenzati e contaminati dalla superstizione - sia Europea che Americana - che cercavano di evangelizzare ${ }^{92}$.

Muratori, comunque, si limitò a criticare gli eccessi della missione gesuitica, critiche che avevano maggiormente a che fare con la realtà religiosa dell'Italia. In realtà, considerando il successo dei Redentoristi nel Regno di Napoli, i suoi commenti posseggono una qualità quasi profetica. Alcuni decenni dopo il suo breve scambio epistolare con Segneri jr, Muratori scrisse, nella sua Della regolata divozion de' Cristiani (1747), che aveva l'intenzione di scoprire la natura della «vera e soda Divozione, distinguendola da quelle Divozioni, che sono superfiziali, e toccando leggermente altre, che hanno apparenza o sostanza di superstizione ${ }^{93}$ ". Nel processo, condannò la devozione gesuitica degli Schiavi di Maria e le processioni del Corpus Domini. Descrisse queste ultime come parate di «Macchine, persone travestite, carri, statue, e grandiosità di comparse», che servivono "solamente a riscuotere dal Popolo ammirazione e cianciumi ${ }^{94}$ ». Eppure, allo stesso tempo, Muratori difendeva devozioni popolari come le medagliette religiose, l'agnus dei, il rosario, gli scapolari, i nastri, le immagini dei santi e i brevi ${ }^{95}$, che tutte insieme avrebbero costituito una parte importante del metodo missionario redentorista che veniva formulato proprio in quel momento.

I missionari gesuiti non erano sordi alle critiche che venivano rivolte loro da parte di Muratori e di altri. Oltre al graduale spostamento verso

${ }^{90}$ Lettere del 20.6 e del 6.7. 1712, M. Campori, a cura di, Epistolario di L.A. Muratori, Modena 1902, iv, p. 1472; cit. da Orlandi, Muratori, cit., 183-84.

${ }^{91}$ Ludovico Antonio Muratori, La vita di P. P. Segneri iuniore, Modena, 1720.

${ }_{92} \mathrm{Cfr}$. Girolamo Imbruglia, Dalle storie dei santi alla storia naturale della religione. L'idea moderna di superstizione, in Rivista storica italiana, CI, 1989, p. 35-84, in particolare, p. $70-8$.

${ }^{93}$ Ludovico Antonio Muratori, Della regolata divozion de' cristiani, $2^{\mathrm{a}}$ ed., Venezia, 1761, p. VI.

${ }_{94}$ Ibidem, p. 379 e 343.

${ }^{95}$ Ibidem, p. 347. 
la campagna, a cui si è fatto riferimento sopra, una lettura delle lettere annuali riguardanti i collegi di Lecce e di Taranto sembra suggerire che i missionari mitigarono le processioni penitenziali dall'ultimo decennio del Seicento in poi. Però questo fatto potrebbe anche indicare - dato che le lettere annuali erano una sintesi delle informazioni mandate a Roma dei lievi cambiamenti in ciò che al centro si riteneva degno di nota circa le attività e i risultati, piuttosto che nel modo di fare le missioni alla periferia ${ }^{96}$. Per provare questa ipotesi si ha bisogno di ulteriori studi sulle relazioni relative alle province italiane della Compagnia che coprano un lungo arco di tempo.

Che cosa possiamo dire a proposito dell'impatto a lungo termine delle missioni? Questa è una domanda a cui è molto più difficile rispondere data la relativa scarsezza di dati. A causa del fatto che nelle relazioni sulle missioni i Gesuiti preferivano dare più rilievo all'aspetto sensazionale piuttosto che a quello più sommesso delle tecniche usate nel missionare, è difficile misurare l'impatto di qualcosa come l'istruzione religiosa sui paesi interessati dalle missioni. Anche se tutte le congregazioni missionarie ponevano l'accento sull'insegnamento del catechismo come parte centrale della missione, sembra invece che sia stato il tono e il carattere delle devozioni e degli esercizi religiosi introdotti a fare un'impressione maggiore sulla gente. Questo era dovuto probabilmente al tipo di insegnamento, che era molto semplice. La conoscenza religiosa aveva un posto secondario rispetto alla corretta pratica religiosa, come abbiamo visto nel paragrafo precedente riguardo alla confessione. L'emotività che caratterizza oggigiorno la pietà popolare nell'Italia meridionale è, per lo meno in parte, il risultato dell'attività missionaria dei Gesuiti e delle altre congregazioni coinvolte nell'opera di «evangelizzazione» nella penisola. Lo strascino, per esempio, era destinato a sopravvivere fino agli anni '50 in pellegrinaggi locali in tutto il Mezzogiorno, molto tempo dopo aver perduto il favore presso altri livelli della società (incluse le stesse autorità ecclesiastiche) ${ }^{97}$.

Altre devozioni sono sopravvissute, ma i loro significati sono stati alterati per essere adattati ai bisogni della cultura contadina. Perciò gli «abitini di Maria", introdotti dai Redentoristi, sono sopravvissuti fino ai nostri giorni come talismani extra-liturgici fatti indossare ai neonati e ai bambini per proteggerli dalla magia malefica ${ }^{98}$. Più significativo, comunque, è stato

${ }^{96}$ L'ultima relazione missionaria giunta fino a noi è del 1687, e quindi le relazioni stesse non possono fare altra luce su questo possibile sviluppo.

${ }^{97}$ Cfr. Annabella Rossi, Le feste dei poveri, 2a ed., Palermo, 1986, p. 75-6.

${ }^{98}$ Ernesto de Martino, Sud e magia, Torino, 1966, p. 36-8. 
l'impatto delle forme penitenziali gesuitiche, di cui alcuni elementi sopravvivono ancora oggi a livello locale in processioni spontanee ed extra-liturgiche di penitenti maschi che si battono a sangue durante la Settimana Santa. Due sono le cose interessanti a questo proposito. Primo, la distinzione di sesso, dal momento che richiama quella originariamente imposta dagli stessi missionari. Secondo, l'«analogia con Cristo» nella sofferenza e nello spargimento di sangue da parte del penitente ha ormai acquisito il significato di rito salvifico e dell'affermazione della forza e della resistenza maschile ${ }^{99}$.

L'impatto delle processioni penitenziali e dei drammi sacri messi in scena dai Gesuiti era nello stesso tempo molto immediato e impressionante, se si tiene conto degli avvenimenti descritti nelle relazioni delle missioni, e anche cumulativo e di più lunga durata, come è dimostrato dalla sua sopravvivenza al di fuori della religione organizzata. Questa ultima caratteristica era già evidente nel decennio che seguì l'espulsione della Compagnia di Gesù dal Regno di Napoli nel 1767. Una prammatica reale emanata nel 1779, quando il clima anti-gesuitico ancora prevaleva, merita di essere riportata per esteso.

Essendo pervenuti a notizia di S[ua] M[aestà] (D[io] G[uardi]) gli scandalosi abusi di taluni del basso Popolo in parecchi luoghi del Regno, che nel Giovedì e nel Venerdì Santo, in vece di onorare la memoria della Passione del nostro Divino Redentore con una vera interna compunzione, e segreto ravvedimento de' proprj falli, la dissonorano piuttosto, e la profanano, per mezzo di varie sceniche comparse, e spettacoli popolareschi; alcuni coll'andar nudi per le piazze, e per le strade, battendosi a sangue; altri con rappresentare i Sagri Misterj della Passione, vestiti, chi da Cristo, e chi da Giudei, e da Manigoldi. Quindi la M[aestà] S[ua] spinto dallo zelo per la Religione, e per ovviare il fanatismo di tali falsi divoti, ha risoluto, che da ora in avanti non vi sia chi ardisca, così in Settimana Santa, come in ogn'altro tempo dell'anno comparir da Battenti, o rappresentare i Misterj della Passione, sotto pena di esser condannata alla frusta ${ }^{100}$.

Benché le autorità secolari ed ecclesiastiche avessero inferto un duro colpo ai Gesuiti, a loro fu lasciato il compito di mettere le cose in ordine dopo la loro partenza. Come si è cercato di dimostrare nel corso di questo

${ }^{99}$ Novi Chavarria, L'attività, cit., p. 181.

100 "Interdictum sacrae passionis mysteriae exprimere», Napoli, 4.5.1779, in Lorenzo Giustiniani, Nuova collezione delle prammatiche del Regno di Napoli, IV, Napoli, 1803-5, p. 276. Può sembrarci strano che la pena per la flagellazione pubblica fosse una frustata parimenti pubblica. 
saggio furono le altre congregazioni missionarie attive nel Regno di Napoli ad essere maggiormente in grado di rinnovare le loro strategie e i loro metodi alla luce delle critiche precedenti, tenendo sempre presente la realtà di una popolazione in gran parte analfabeta e misera. Il dolce amore della Madonna veniva messo in primo piano, mentre veniva attenuata la minaccia della dannazione eterna e il bisogno costante di lugubri atti di penitenza.

Queste missioni, che potremmo chiamare post-gesuitiche, tentavano innanzittutto di lavorare in armonia con le autorità locali, sia quelle ecclesiastiche che quelle secolari. Per molto tempo i vescovi avevano impiegato le missioni per rinforzare la qualità dell'osservanza religiosa nelle loro diocesi, come quando il vescovo di Lecce Fabrizio Pignatelli fece richiesta di missioni gesuitiche successivamente all'Interdetto del 1711-1910. Ma i Redentoristi agirono anche come forza in favore della calma e della stabilità sociale. I vescovi fecero richiesta di missioni redentoriste direttamente al re, che concesse loro un modesto contributo finanziario, mentre allo stesso tempo utilizzò la missione per ristabilire l'ordine e la pace sociale subito dopo i disordini. L'opera dei missionari era perciò sempre più richiesta durante i moti rivoluzionari che cominciarono nel 1799. In quell'anno il vescovo di Oria Fabrizio Cimino chiese il permesso per le missioni e ottenne otto preti redentoristi che andarono in giro per la diocesi per sei mesi, predicando la parola di Dio, l'obbedineza alle autorità ecclesiastiche e secolari, il rispetto per la corona e le sue leggi, e la pace tra la gente ${ }^{102}$. Questa attività fu accentuata dopo la Restaurazione. Questo periodo vide la formazione di una nuova congregazione missionaria, la Congregazione del Preziosissimo Sangue. Fondata in linea con i Redentoristi, ma rinunciando alle loro infiammanti prediche barocche, contribuì "all'evoluzione verso una pietà più calda, più popolare, e anche più facile, più esteriore, che è una manifestazione di religiosità presente naturalmente fin dal Sei-Settecento, ma che nell'Ottocento diventa prevalente ${ }^{103}$ ».

Sia che lo stile di predicare fosse teatrale ed esteriorizzato, come quello dei Gesuiti, dei Missionari apostolici e dei Redentoristi, sia che fosse

${ }^{101}$ Giovanni Barrella, La Compagnia di Gesù nelle Puglie, Lecce, 1941, p. 79 , n. 12.

${ }^{102}$ Carmelo Turrisi, La diocesi di Oria nell'Ottocento, Roma, 1978, p. 331-2.

${ }^{103}$ Guido Verucci, Chiesa e società nell'Italia della Restaurazione (1814-1830), in Rivista di storia della Chiesa in Italia, XXX, 1976, p. 25-72, a p. 61. 
più sobrio, come quello dei Lazzaristi, tutte le congregazioni missionarie condividevano una caratteristica di base : la semplicità e l'immediatezza del messaggio. Essi consideravano la predica come uno strumento utile per instillare nei fedeli la paura di Dio e della dannazione eterna, ritenuta necessaria per portarli al pentimento e a una sincera e completa confessione. Nelle varie strategie che abbiamo preso in considerazione, alla predica si accompagnava l'introduzione e la diffusione di devozioni tipicamente barocche, per tenere la gente sulla retta via. La persistenza, fino a oggi, di tali pratiche religiose nell'Italia meridionale è testimonianza del contributo dato dalle missioni, così come dei loro limiti. La stessa cosa vale anche per le Americhe, dove l'«idolatrizzazione» di oggetti devozionali cristiani da parte delle popolazioni indigene accompagnò la colonizzazione culturale svolta dai missionari. Su entrambi i continenti l'introduzione di devozioni si rivelò una tecnica missionaria efficace, anche in zone dove il clero locale era ancora negligente nei suoi doveri pastorali e non forniva un esempio adeguato.

I missionari nel Regno di Napoli, come era il caso altrove, si accontentavano prevalentemente di assicurare la pratica corretta delle norme della Chiesa. I loro risultati, almeno a questo riguardo, davano loro una ragione di soddisfazione ${ }^{104}$. Ma i tentativi dei missionari di "accomodarsi alla capacità del popolo", in modo da non confonderlo o da non far sorgere dubbi nella sua mente, richiedevano una semplicità che rasentava la superficialità. Il messaggio che ne scaturiva metteva in primo piano la pratica religiosa piuttosto che la conoscenza religiosa, i poteri miracolosi piuttosto che la meditazione spirituale. A causa del contenuto miracolistico del messaggio e del modo in cui veniva presentato, le forme devozionali erano accettate e assimilate, benchè spogliate del loro contesto dottrinale.

In alcuni casi le strategie delle missioni venivano addirittura trasformate per adattarle al sistema rituale locale, secondo un processo di negoziazione tra differenti livelli di cultura. Come abbiamo visto, la sola sacra presenza dei missionari poteva essere più significativa dell'esempio religioso che essi offrivano. Ciò nonostante, l'impatto e gli effetti complessivi delle missioni non sono qualitativamente molto diversi dal messaggio che gli stessi missionari si adoperarono di presentare nel corso di diversi secoli. Si potrebbe dire perciò che i missionari abbiano raggiunto i loro scopi di base. Per quanto concerne gli effetti duraturi dell'istruzione religiosa è più

${ }^{104}$ Croix, Bretagne, cit., II, p. 1240. 
difficile giungere ad una conclusione. Ma, poiché una missione durava solo alcune settimane per volta, e poiché si metteva in risalto la conversione e il pentimento, non potevano essere molto profondi. Fu solo nell'Ottocento che il clero locale fu in grado di assumersi l'incarico e la responsabilità di impartire una semplice istruzione religiosa, e questo fu in gran parte dovuto alla preparazione data loro dai missionari. 